\title{
COOPERATIVE AUTOMATIC CONTROL FOR THE CANOPY POSTURE OF A FOUR-LEG HYDRAULIC SUPPORT
}

\author{
$\mathrm{Hu}, \mathrm{X} . \mathrm{P} .{ }^{*}, * *$ \\ * School of Mechanical Electronic \& Information Engineering, China University of Mining \& \\ Technology-Beijing, Beijing 100083, China \\ ${ }^{* * *}$ Institute of Intelligent Mines and Robotics, China University of Mining \& Technology-Beijing, \\ Beijing 100083, China \\ E-Mail: huxiangpeng321@163.com
}

\begin{abstract}
The contact state between the canopy and roof of a hydraulic support considerably influences the bearing capacity and stability of the support. To ensure a secure contact between the canopy and roof in the setting stage, this paper proposes a cooperative double closed-loop automatic control technique for the canopy posture, with MATLAB used to simulate the canopy posture under different roof conditions. First, a cooperative double closed-loop automatic control model was established. Second, the method to determine the target value of the canopy posture and constraint conditions of the rising process were formulated. Finally, by simulating the canopy posture under three roof conditions, the response curve of the canopy posture and the leg length were obtained. The results show that the front and rear legs can rise through either synchronous or asynchronous coordination, and the canopy posture can approach the target value. The presented findings can guide the realization of the automatic control of the canopy posture of hydraulic support.

(Received in August 2020, accepted in November 2020. This paper was with the authors 2 weeks for 1 revision.)
\end{abstract}

Key Words: Four-Leg Hydraulic Support, Canopy Posture, Cooperative Control, Double Closed-Loop

\section{INTRODUCTION}

The canopy posture affects not only the bearing capacity and stability of a hydraulic support, but also the corresponding support to the roof [1]. The coupling state between the support and roof is ideal when the canopy is in full contact with the roof $[2,3]$. Enhancing the posture monitoring of the hydraulic support and automatic posture control can help realize the intelligence of the working surface [4].

According to the bearing characteristics, the operation of a hydraulic support can be divided into a setting stage, increasing resistance stage and constant resistance stage. The main goals of the setting stage are to ensure that the canopy is in secure contact with the roof and to attain the value of the setting load. However, with the advance of working surface, the state of roof also changes. The current control method of support legs cannot ensure that the canopy and roof are in ideal contact, and it is easy for the front or rear toe of the canopy to contact the roof first, and then it is easy to cause the instability of the support and deteriorate the force on the support. Therefore, the use of an automatic control technology for the canopy posture to ensure the ideal contact between the canopy and roof in the setting stage can help enhance the bearing capacity of the support in the increasing and constant resistance stages and maintain the roof stability.

\section{RELATED WORKS}

Many scholars have examined approaches to realize the monitoring and control of the canopy posture. Lin and Miao [5] established a relationship between the support height, deflection angle of the canopy, and driving rams. Liang et al., Cui, Chen and Li, and Asl and Han [6-9] determined the canopy posture through a multi-sensor contact and visual measurement method. Kim and Kim, and Nair and Ezhilarasi $[10,11]$ adopted EMC methods to study robot motion 
control problems. Liu et al. and Yang et al. [12,13] used ADAMS and AMESim to study coordinated simulation of hydraulic pump. Liang et al. [14] expressed the change in the length of the legs and balance rams in terms of the corresponding equivalent stiffness and pressure. Zhang et al. [15] developed a hydraulic support position and posture detection device, based on the principle of three noncolinear points. Yang et al. [16] used LiDAR to measure the posture of the hydraulic support relative to an inspection robot and proposed relative pose calculation formulas. Ren et al. [17] used a radial basis function neural network controller to control the canopy posture by controlling the length of the balance ram and legs. Meng et al. $[18,19]$ applied a multi-software co-simulation method to realize the automatic control of the support posture. Xie et al. and Ge et al. [20,21] developed a method to virtually adjust the support posture of support groups in the propulsion state.

Although these studies provided notable guidance to realize the automatic control of the canopy posture, the uniqueness of the relationship between the canopy posture and driving rams was not examined. Moreover, the studies were focused on realizing the adjustment and simulation of the canopy posture, and the determination of the target canopy posture was not considered. In addition, although certain novel controllers can realize the automatic control of the canopy posture, the existing mature controllers of the support are not employed, which limits the engineering application of such approaches. In general, the built-in displacement sensor used in an advancing ram can achieve a satisfactory control accuracy [22].

Considering the existing studies and focusing on a four-leg support, a cooperative double closed-loop control method for the canopy posture was developed in this study, and the canopy posture was simulated under different roof conditions by using the MATLAB. The objectives were to ensure a secure contact between the canopy and floor in the setting stage and to provide a novel solution to realize the automatic control of the canopy posture.

The remaining paper is organized as follows. Section 3 describes the simulation model for the collaborative double closed-loop automatic control of the canopy posture. Moreover, the functions of each module are presented, and the one-to-one mapping relationship between the canopy posture and driving rams is explained, along with the method to determine the target canopy posture and constraints of the rising process. Section 4 presents the canopy posture response curves under the three roof conditions. Section 5 presents the concluding remarks.

\section{METHODOLOGY}

\subsection{Logic and core modules of automatic control of the canopy posture}

The cooperative double closed-loop automatic control logic for the canopy posture is shown in Fig. 1. The proposed logic is divided into four main modules and two sub-modules. Considering the 'fall-move-rise' configuration of the support as an action cycle, the function of module 1 is to determine the canopy posture reference, as a reference for the target posture of the rise motion. Before executing the cycle, that is, before descending the support, the base deflection angle and length of the front and rear legs are determined through the base inclination sensor and leg stroke sensor, respectively. Subsequently, the sub-module programme for the one-toone mapping of the canopy posture and driving rams is invoked to convert the driving space to the pose space to obtain the reference datum for the canopy posture. Module 2 is used to determine the target value of the canopy posture, determine the base deflection angle after moving the support, and invoke the target height sub-module programme according to the canopy posture reference to obtain the target value of the canopy posture of the rising motion, that is, the height and deflection angle. Subsequently, the sub-module programme for the oneto-one mapping of the canopy posture and driving rams is invoked again to convert the pose space to the driving space to obtain the front and rear leg lengths corresponding to the target value of the canopy posture. Modules 3 and 4 are used to implement the coordinated control of 
the front and rear legs. The front and rear leg lengths, obtained using module 2, are used as the expected output of the closed-loop control. Furthermore, the coordinated action of the front and rear legs is predicted through the constraint equations and fed back to the controller to determine the cooperative action of the front and rear legs. Sub-module I is used to establish the one-toone mapping of the canopy posture and driving rams to realize the mutual conversion between the pose and driving spaces. Sub-module II is used to determine the target height of the canopy posture of the rising motion through the advancing mechanism.
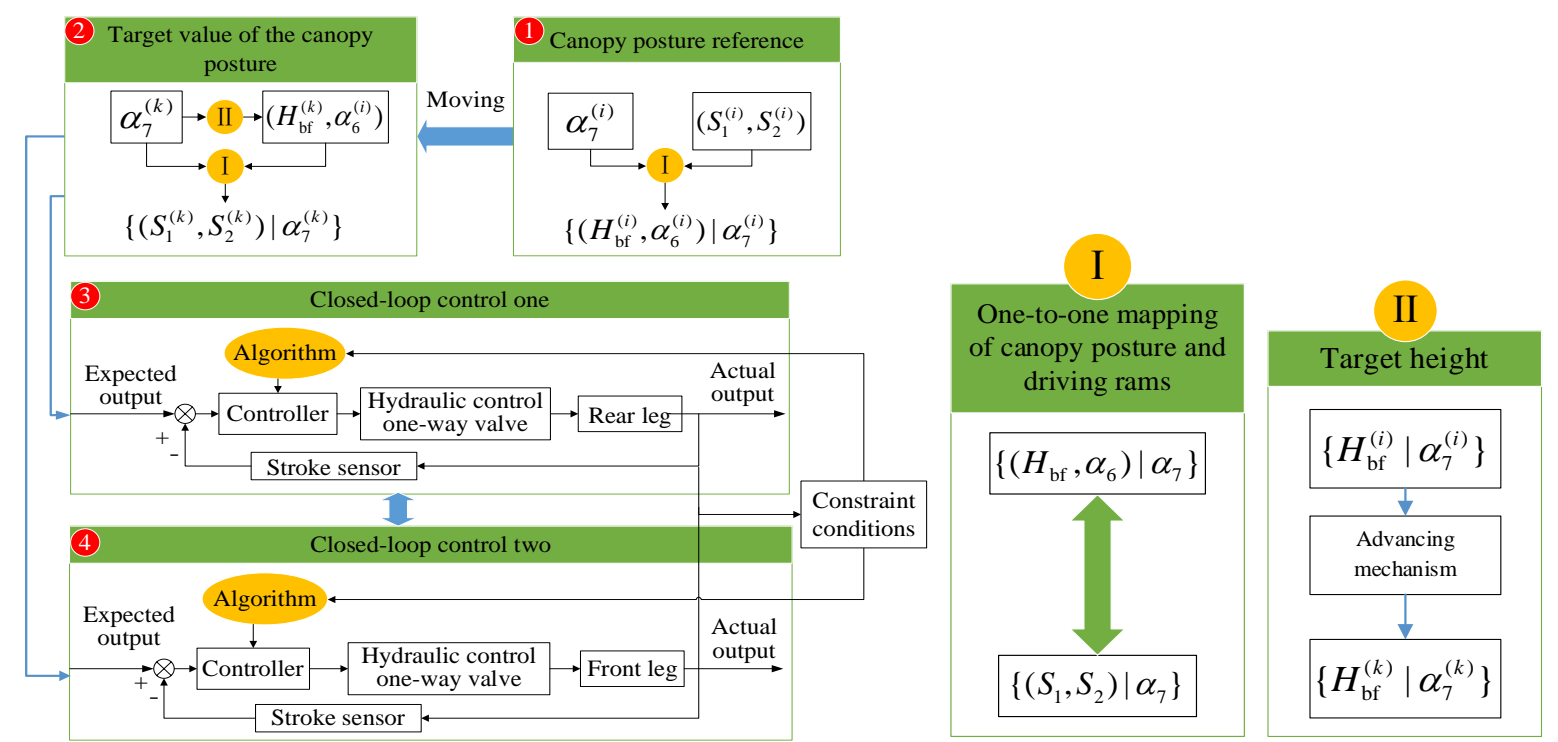

Figure 1: Cooperative double closed-loop automatic control logic for the canopy posture.

\subsection{One-to-one mapping of the canopy posture and driving rams}

The kinematics model of the four-leg support is shown in Fig. 2. A coordinate system $\{x O y\}$ is established, with point $O$ at the toe of the base considered as the coordinate origin.

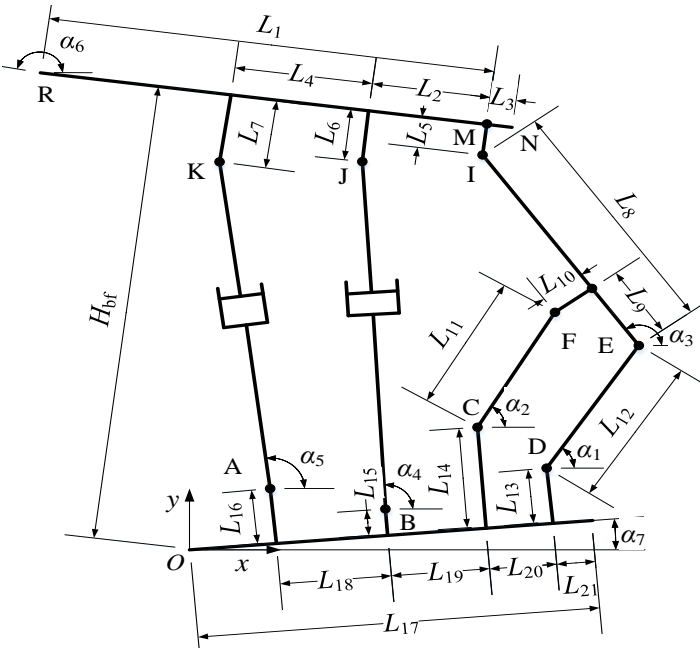

Figure 2: Kinematic model of the four-leg support.

Considering the three closed vector rings of CDEF, ABJK and ADEIK, the following three complex equations can be obtained:

$$
\left\{\begin{array}{l}
L_{12} e^{j \alpha_{1}}+L_{\mathrm{EF}} e^{j \theta_{1}}=L_{\mathrm{DC}} e^{j \theta_{2}}+L_{11} e^{j \alpha_{2}} \\
S_{2} e^{j \alpha_{4}}+L_{\mathrm{JK}} e^{j \theta_{3}}=L_{\mathrm{BA}} e^{j \theta_{4}}+S_{1} e^{j \alpha_{5}} \\
L_{\mathrm{AD}} e^{j \theta_{5}}+L_{12} e^{j \alpha_{1}}+L_{8} e^{j \alpha_{3}}+L_{\mathrm{IK}} e^{j \theta_{6}}=S_{1} e^{j \alpha_{5}}
\end{array}\right.
$$


where, $L_{1} \sim L_{21}$ are the support structure parameters; $S_{1}$ and $S_{2}$ denote the front and rear leg length, respectively; $L_{\mathrm{EF}}$ is the distance from the upper hinge point of the rear linkage to that of the front linkage; $L_{\mathrm{DC}}$ is the distance from the lower hinge point of the rear linkage to that of the front linkage; $L_{\mathrm{JK}}$ is the distance from the upper hinge point of the rear leg to that of the front leg; $L_{\mathrm{BA}}$ is the distance from the lower hinge point of the rear leg to that of the front leg; $L_{\mathrm{AD}}$ is the distance from the lower hinge point of the front leg to that of the rear linkage; $L_{\mathrm{IK}}$ is the distance from the hinge point between the canopy and goaf shield to the upper hinge point of the front leg; $\alpha_{1}, \alpha_{2}, \alpha_{3}, \alpha_{4}, \alpha_{5}$, and $\alpha_{6}$ denote the angles between the $x$-axis and the rear linkage, front linkage, goaf shield, rear leg, front leg, and canopy, respectively; $\alpha_{7}$ is the base deflection angle; $\theta_{1}, \theta_{2}, \theta_{3}, \theta_{4}, \theta_{5}$, and $\theta_{6}$ denote the angle between the $x$-axis and $L_{\mathrm{EF}}, L_{\mathrm{DC}}, L_{\mathrm{JK}}$, $L_{\mathrm{BA}}, L_{\mathrm{AD}}$, and $L_{\mathrm{IK}}$, respectively. $\gamma_{1}$ is the angle between $L_{\mathrm{EF}}$ and the goaf shield. $\gamma_{2}$ is the angle between $L_{\mathrm{DC}}$ and the base. $\gamma_{3}$ is the angle between $L_{\mathrm{JK}}$ and the canopy. $\gamma_{4}$ is the angle between $L_{\mathrm{BA}}$ and the base. $\gamma_{5}$ is the angle between $L_{\mathrm{AD}}$ and the base. $\gamma_{6}$ is the angle between $L_{\mathrm{IK}}$ and the canopy.

$$
\begin{array}{lll}
L_{\mathrm{EF}}=\sqrt{L_{9}^{2}+L_{10}^{2}}, & L_{\mathrm{DC}}=\sqrt{L_{20}^{2}+\left(L_{14}-L_{13}\right)^{2}}, & \theta_{1}=\alpha_{3}+\gamma_{1}, \\
L_{\mathrm{JK}}=\sqrt{L_{4}^{2}+\left(L_{7}-L_{6}\right)^{2},} & L_{\mathrm{BA}}=\sqrt{L_{18}^{2}+\left(L_{16}-L_{15}\right)^{2}}, & \theta_{2}=\pi-\left(\gamma_{2}-\alpha_{7}\right), \\
L_{\mathrm{AD}}=\sqrt{\left(L_{18}+L_{19}+L_{20}\right)^{2}+\left(L_{13}-L_{16}\right)^{2}}, & L_{\mathrm{IK}}=\sqrt{\left(L_{2}+L_{4}\right)^{2}+\left(L_{7}-L_{5}\right)^{2}}, & \theta_{3}=\alpha_{6}+\gamma_{3}, \\
\theta_{4}=\pi-\left(\gamma_{4}-\alpha_{7}\right), & \theta_{5}=\alpha_{7}+\gamma_{5}, & \theta_{6}=\alpha_{6}+\gamma_{6}, \\
\gamma_{1}=\arctan \left(\frac{L_{10}}{L_{9}}\right), & \gamma_{2}=\arctan \left(\frac{L_{14}-L_{13}}{L_{20}}\right), & \gamma_{3}=\arctan \left(\frac{L_{7}-L_{6}}{L_{4}}\right), \\
\gamma_{4}=\arctan \left(\frac{L_{16}-L_{15}}{L_{18}}\right), & \gamma_{5}=\arctan \left(\frac{L_{13}-L_{16}}{L_{18}+L_{19}+L_{20}}\right), & \gamma_{6}=\arctan \left(\frac{L_{7}-L_{5}}{L_{2}+L_{4}}\right) .
\end{array}
$$

Eq. (1) can be expanded using Euler's formula to obtain the following six nonlinear equations:

$$
\left\{\begin{array}{l}
f_{1}=L_{12} \cos \alpha_{1}+L_{\mathrm{EF}} \cos \theta_{1}-L_{\mathrm{DC}} \cos \theta_{2}-L_{11} \cos \alpha_{2} \\
f_{2}=L_{12} \sin \alpha_{1}+L_{\mathrm{EF}} \sin \theta_{1}-L_{\mathrm{DC}} \sin \theta_{2}-L_{11} \sin \alpha_{2} \\
f_{3}=S_{2} \cos \alpha_{4}+L_{\mathrm{JK}} \cos \theta_{3}-L_{\mathrm{BA}} \cos \theta_{4}-S_{1} \cos \alpha_{5} \\
f_{4}=S_{2} \sin \alpha_{4}+L_{\mathrm{JK}} \sin \theta_{3}-L_{\mathrm{BA}} \sin \theta_{4}-S_{1} \sin \alpha_{5} \\
f_{5}=L_{\mathrm{AD}} \cos \theta_{5}+L_{12} \cos \alpha_{1}+L_{8} \cos \alpha_{3}+L_{\mathrm{IK}} \cos \theta_{6}-S_{1} \cos \alpha_{5} \\
f_{6}=L_{\mathrm{AD}} \sin \theta_{5}+L_{12} \sin \alpha_{1}+L_{8} \sin \alpha_{3}+L_{\mathrm{IK}} \sin \theta_{6}-S_{1} \sin \alpha_{5}
\end{array}\right.
$$

Given $\alpha_{7}, S_{1}$ and $S_{2}$, the six unknowns $\alpha_{1}, \alpha_{2}, \alpha_{3}, \alpha_{4}, \alpha_{5}$ and $\alpha_{6}$ can be obtained using Eq. (2), and the linear equation on the upper surface of canopy can be expressed as follows:

$$
\begin{array}{cll}
\text { where, } k=\tan \alpha_{6}, & x_{\mathrm{M}}=x_{\mathrm{I}}+L_{5} \cos \left(\alpha_{6}-\pi / 2\right), & y_{\mathrm{M}}=y_{\mathrm{I}}+L_{5} \sin \left(\alpha_{6}-\pi / 2\right), \\
x_{\mathrm{I}}=x_{\mathrm{E}}-L_{8} \cos \left(\pi-\alpha_{3}\right), & y_{\mathrm{I}}=y_{\mathrm{E}}-L_{8} \sin \left(\pi-\alpha_{3}\right), & x_{\mathrm{E}}=x_{\mathrm{D}}+L_{12} \cos \alpha_{1}, \\
y_{\mathrm{E}}=y_{\mathrm{D}}+L_{12} \sin \alpha_{1}, & x_{\mathrm{D}}=L_{\mathrm{OD}} \cos \left(\alpha_{7}+\gamma_{7}\right), & y_{\mathrm{D}}=L_{\mathrm{OD}} \sin \left(\alpha_{7}+\gamma_{7}\right), \\
L_{\mathrm{OD}}=\sqrt{\left(L_{17}-L_{21}\right)^{2}+L_{13}^{2},} & \gamma_{7}=\arctan \left(\frac{L_{13}}{L_{17}-L_{21}}\right) . &
\end{array}
$$

$L_{O \mathrm{D}}$ is the distance from point $O$ to the lower hinge point of the rear leg. $\gamma_{7}$ is the angle between $L O D$ and the base.

The support height $H_{\mathrm{bf}}$ is defined as the vertical distance from point $O$ to the canopy surface, that is, the distance from point $O$ to the straight line on the canopy surface. Substituting $O(0,0)$ into Eq. (3), the support height can be obtained as follows:

$$
H_{\mathrm{bf}}=\frac{\left|y_{\mathrm{M}}-k x_{\mathrm{M}}\right|}{\sqrt{k^{2}+1}}
$$


$H_{\mathrm{bf}}$ is a function of $\alpha_{1}, \alpha_{3}, \alpha_{6}$ and $\alpha_{7}$. When $\alpha_{7}, S_{1}$ and $S_{2}$ are known, the seven unknowns $\alpha_{1}$, $\alpha_{2}, \alpha_{3}, \alpha_{4}, \alpha_{5}, S_{1}$ and $S_{2}$ can be obtained in combination with Eq. (2). Therefore, the one-to-one mapping of the canopy posture and driving rams can be expressed as: $\left\{\left(S_{1}, S_{2}\right) \mid \alpha_{7}\right\} \leftrightarrow\left\{\left(H_{\mathrm{bf}}, \alpha_{6}\right) \mid \alpha_{7}\right\}$.

\subsection{Canopy posture reference and its target value}

The value of the canopy posture before the 'fall-move-rise' cycle of the support, that is, the canopy posture before the descend of the support cannot be used as the target value for the rising motion, because the roof may be inclined, or the base posture may have changed after the falling and moving motions. To determine the target value of the canopy posture under the rising of the given cycle, the following assumptions are considered:

1) The hinge point of the advancing rod and scraper conveyor is considered as the origin of the absolute coordinate system $\left\{x O_{0} y\right\}$, as shown in Fig. 3.

2) When the canopy and floor fit along a straight line, and the floor state remains unchanged before and after the movement, the straight line is the canopy posture reference line for the rising in the given cycle, as shown in Fig. 4.
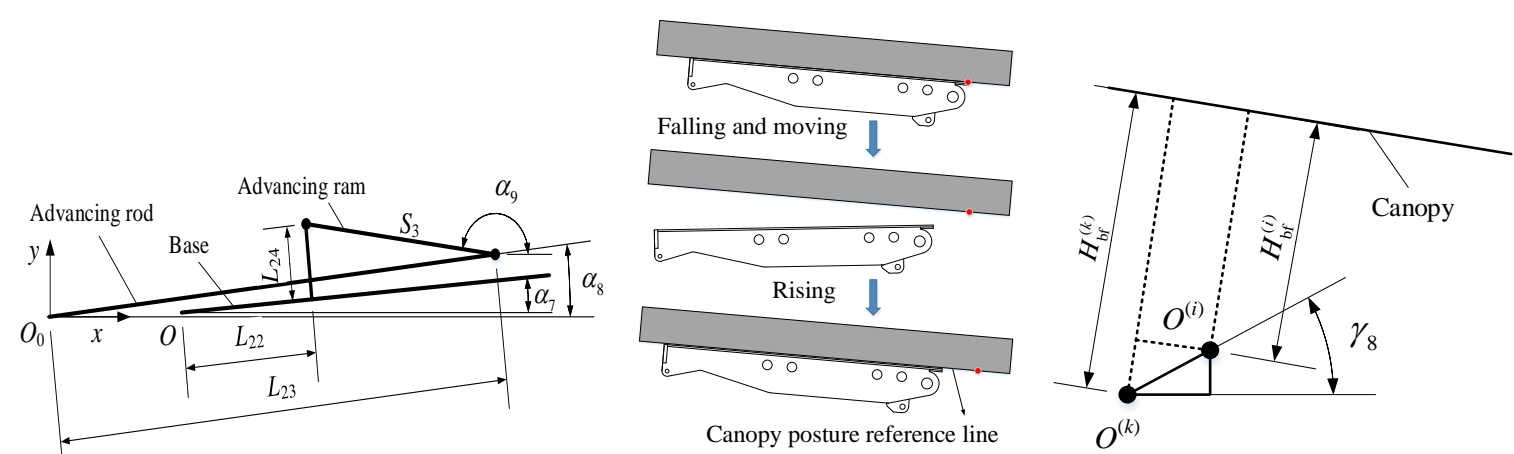

Figure 3: Absolute coordinate system. Figure 4: Canopy posture Figure 5: Height relationship before reference line. and after the movement.

The canopy posture before the support descends is indicated using the superscript $(i)$, and the coordinate system is marked as $\left\{x O^{(i)} y\right\}$. The target value of rising in this cycle is marked using the superscript $(k)$, and the coordinate system is marked as $\left\{x O^{(k)} y\right\}$. The reference line slope $k=\tan \alpha_{6}^{(i)}$ can be obtained considering the canopy posture $\left\{\left(H_{\mathrm{bf}}^{(i)}, \alpha_{6}^{(i)}\right) \mid \alpha_{7}^{(i)}\right\}$ before moving, and the reference line equation can then be obtained by substituting this value into Eq. (3).

$$
y-y_{\mathrm{M}}^{(i)}=k\left(x-x_{\mathrm{M}}^{(i)}\right)
$$

Therefore, the support height before the movement can be obtained considering Eq. (4), as follows:

$$
\left.H_{\mathrm{bf}}^{(i)}=\left(\mid y_{\mathrm{M}}^{(i)}-k x_{\mathrm{M}}^{(i)}\right) \mid\right) / \sqrt{k^{2}+1}
$$

The relationship between the support target height $H_{\mathrm{bf}}^{(k)}$ and height $H_{\mathrm{bf}}^{(i)}$ before and after the movement, respectively, is as follows, as indicated in Fig. 5:

$$
H_{\mathrm{bf}}^{(k)}=H_{\mathrm{bf}}^{(i)}+O^{(i)} O^{(k)} \cos \left(\alpha_{6}^{(i)}-\pi / 2-\gamma_{8}\right)
$$

where, $O^{(i)} O^{(k)}$ is the distance between the toe of the base before and after the support moves. $\gamma_{8}$ is the angle between $O^{(i)} O^{(k)}$ and $x$-axis; the angle is positive and negative when the toe of the base sinks and lifts, respectively.

$$
O^{(i)} O^{(k)}=\sqrt{\left(x_{O_{0}^{(i)}}-x_{O_{0}^{(k)}}\right)^{2}+\left(y_{O_{0}^{(i)}}-y_{O_{0}^{(k)}}\right)^{2}},
$$

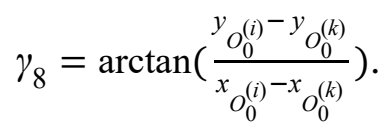


The coordinates $\left(x_{O_{0}^{(i)}}, y_{O_{0}^{(i)}}\right)$ and $\left(x_{O_{0}^{(k)}}, y_{O_{0}^{(k)}}\right)$ are relative to $\left\{x O_{0} y\right\}$ and can be determined using the following equations:

$$
\left\{\begin{array}{l}
x_{O_{0}^{(i)}}=L_{23} \cos \alpha_{8}^{(i)}-S_{3}^{(i)} \cos \left(\pi-\alpha_{9}^{(i)}\right)+L_{24} \cos \alpha_{7}^{(i)}-L_{22} \cos \alpha_{7}^{(i)} \\
y_{O_{0}^{(i)}}=L_{23} \sin \alpha_{8}^{(i)}-S_{3}^{(i)} \sin \left(\pi-\alpha_{9}^{(i)}\right)+L_{24} \sin \alpha_{7}^{(i)}-L_{22} \sin \alpha_{7}^{(i)} \\
x_{O_{0}^{(k)}}=L_{23} \cos \alpha_{8}^{(k)}-S_{3}^{(k)} \cos \left(\pi-\alpha_{9}^{(k)}\right)+L_{24} \cos \alpha_{7}^{(k)}-L_{22} \cos \alpha_{7}^{(k)} \\
y_{O_{0}^{(k)}}=L_{23} \sin \alpha_{8}^{(k)}-S_{3}^{(k)} \sin \left(\pi-\alpha_{9}^{(k)}\right)+L_{24} \sin \alpha_{7}^{(k)}-L_{22} \sin \alpha_{7}^{(k)}
\end{array}\right.
$$

where, $L_{22}$ is the distance from point $O$ to the hinge point of the advancing ram and base, along the direction of the base. $L_{23}$ is the advancing rod length. $L_{24}$ is the vertical distance from the hinge point of the advancing ram and base to the base. $S_{3}^{(i)}$ and $S_{3}^{(k)}$ denote the advancing ram lengths before and after the movement, respectively. $\alpha_{8}^{(i)}$ and $\alpha_{8}^{(k)}$ denote the angles between the advancing rod and $x$-axis, and $\alpha_{9}^{(i)}$ and $\alpha_{9}^{(k)}$ denote the angles between the advancing ram and $x$ axis.

Thus, the target value of the canopy posture is $\left\{\left(H_{\mathrm{bf}}^{(k)}, \alpha_{6}^{(i)}\right) \mid \alpha_{7}^{(k)}\right\}$ in this cycle. In this case, the target length $\left\{\left(S_{1}^{(k)}, S_{2}^{(k)}\right) \mid \alpha_{7}^{(k)}\right\}$ of the front and rear legs can be obtained through the one-toone mapping of the canopy posture and driving rams.

\subsection{Constraint conditions}

When the front toe of a canopy is connected to the floor first, the support may rotate easily, as shown in Fig. 6 a. When the rear toe of the canopy is connected to the floor first, as the rising continues, the support may repeatedly impact the floor, as shown in Fig. 6 b. The ideal contact state of the canopy and floor corresponds to a complete contact, as shown in Figs. $6 \mathrm{c}-\mathrm{e}$. To avoid the situation shown in Figs. 6 a and b, a certain constraint condition must be introduced: During the rising motion, the height of the front and rear toes of the canopy are smaller than the height of the intersection point of the straight line perpendicular to the $x$-axis at this point and the canopy posture reference line, as shown in Fig. 7.

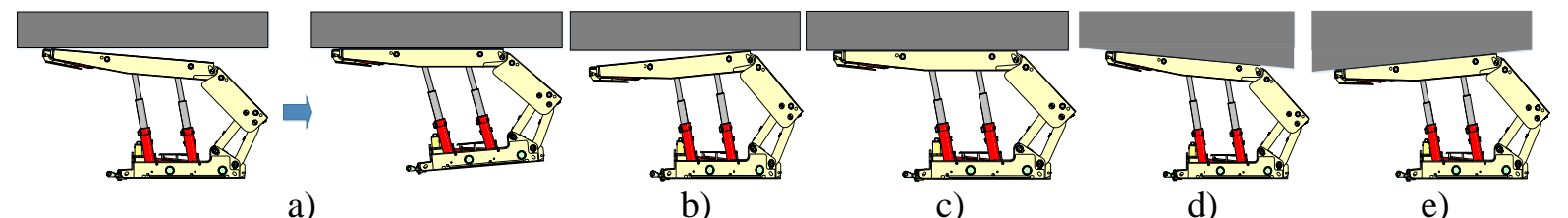

Figure 6: State of the contact between the canopy and roof.

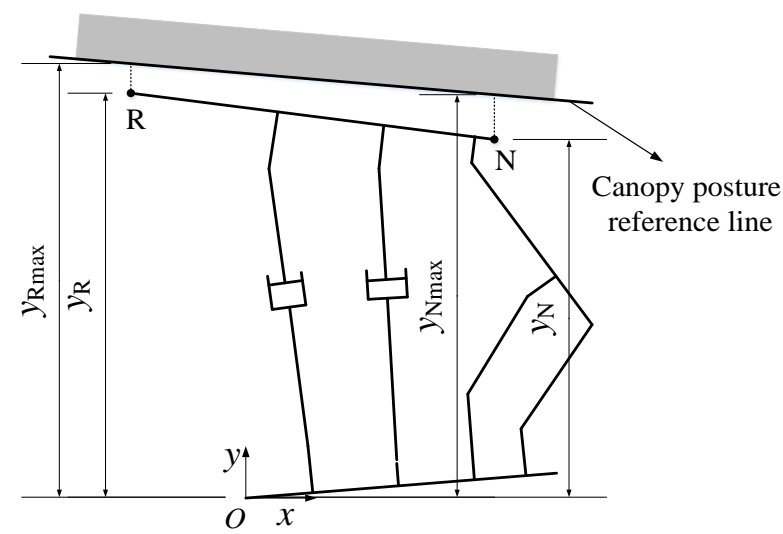

Figure 7: Schematic of the constraint conditions. 
The constraint conditions can be expressed as follows:

$$
\left\{\begin{array}{l}
y_{\mathrm{R}}^{(j)} \leq y_{\mathrm{Rmax}}^{(j)} \\
y_{\mathrm{N}}^{(j)} \leq y_{\mathrm{Nmax}}^{(j)}
\end{array}\right.
$$

where, $y_{\mathrm{R}}^{(j)}, y_{\mathrm{Rmax}}^{(j)}, y_{\mathrm{N}}^{(j)}$ and $y_{\mathrm{Nmax}}^{(j)}$ denote the ordinates at time $j$ during the rising motion.

During the rising process, the coordinates of points $\mathrm{R}$ and $\mathrm{N}$ at the front and rear toes of the canopy, respectively, are as follows:

$$
\left\{\begin{array}{l}
x_{\mathrm{R}}^{(j)}=x_{\mathrm{M}}^{(j)}-L_{1} \cos \left(\pi-\alpha_{6}^{(j)}\right) \\
y_{\mathrm{R}}^{(j)}=y_{\mathrm{M}}^{(j)}+L_{1} \sin \left(\pi-\alpha_{6}^{(j)}\right) \\
x_{\mathrm{N}}^{(j)}=x_{\mathrm{M}}^{(j)}+L_{3} \cos \left(\pi-\alpha_{6}^{(j)}\right) \\
y_{\mathrm{N}}^{(j)}=y_{\mathrm{M}}^{(j)}-L_{3} \cos \left(\pi-\alpha_{6}^{(j)}\right)
\end{array}\right.
$$

Using Eq. (3), the straight lines perpendicular to the $x$-axis through points $\mathrm{R}$ and $\mathrm{N}$ can be considered to obtain the following two sets of equations:

$$
\begin{aligned}
& \left\{\begin{array}{l}
y-y_{\mathrm{M}}^{(k)}=k\left(x-x_{\mathrm{M}}^{(k)}\right) \\
x=x_{\mathrm{R}}^{(j)}
\end{array}\right. \\
& \left\{\begin{array}{l}
y-y_{\mathrm{M}}^{(k)}=k\left(x-x_{\mathrm{M}}^{(k)}\right) \\
x=x_{\mathrm{N}}^{(j)}
\end{array}\right.
\end{aligned}
$$

where, $x_{\mathrm{M}}^{(k)}$ and $y_{\mathrm{M}}^{(k)}$ are the coordinates of point $\mathrm{M}$ with respect to $\left\{x O^{(k)} y\right\}$.

$x_{\mathrm{M}}^{(k)}$ and $y_{\mathrm{M}}^{(k)}$ can be obtained through a coordinate transformation:

$$
\left[\begin{array}{l}
x_{\mathrm{M}}^{(k)} \\
y_{\mathrm{M}}^{(k)}
\end{array}\right]=\left[\begin{array}{ll}
1 & 0 \\
0 & 1
\end{array}\right]\left[\begin{array}{l}
x_{\mathrm{M}}^{(i)} \\
y_{\mathrm{M}}^{(i)}
\end{array}\right]+O^{(i)} O^{(k)}\left[\begin{array}{c}
\sin \gamma_{8} \\
\cos \gamma_{8}
\end{array}\right]
$$

According to Eqs. (11) and (12), $y_{\mathrm{Rmax}}^{(j)}$ and $y_{\mathrm{Nmax}}^{(j)}$ can be obtained as follows:

$$
\left\{\begin{array}{l}
y_{\mathrm{R} \max }^{(j)}=k\left(x_{\mathrm{R}}^{(j)}-x_{\mathrm{M}}^{(k)}\right)+y_{\mathrm{M}}^{(k)} \\
y_{\mathrm{N} \max }^{(j)}=k\left(x_{\mathrm{N}}^{(j)}-x_{\mathrm{M}}^{(k)}\right)+y_{\mathrm{M}}^{(k)}
\end{array}\right.
$$

\subsection{Reference, initial, and target values under three conditions}

With the ZZ22000/29/60D four-leg support considered as an example, the support structure parameters are listed in Table I. By substituting the reference value before the movement, base deflection angle after the movement, and change in the coordinates of point $\mathrm{O}$ into Eq. (7), the target height of the canopy posture can be obtained. Subsequently, in combination with the oneto-one mapping of the canopy posture and driving rams, the target lengths of the front and rear legs can be obtained, as shown in Table II.

Table I: Support structure parameters (mm).

\begin{tabular}{|c|c|c|c|c|c|c|c|c|c|c|c|}
\hline$L_{1}$ & 8,015 & $L_{5}$ & 350 & $L_{9}$ & 705 & $L_{13}$ & 510 & $L_{17}$ & 3,681 & $L_{21}$ & 230 \\
\hline$L_{2}$ & 640 & $L_{6}$ & 475 & $L_{10}$ & 25 & $L_{14}$ & 1,295 & $L_{18}$ & 970 & $L_{22}$ & 1,021 \\
\hline$L_{3}$ & 240 & $L_{7}$ & 475 & $L_{11}$ & 2,500 & $L_{15}$ & 340 & $L_{19}$ & 520 & $L_{23}$ & 3,786 \\
\hline$L_{4}$ & 1,660 & $L_{8}$ & 3,255 & $L_{12}$ & 2,405 & $L_{16}$ & 460 & $L_{20}$ & 1,000 & $L_{24}$ & 615 \\
\hline
\end{tabular}


Table II: Reference, initial, and target values under three conditions.

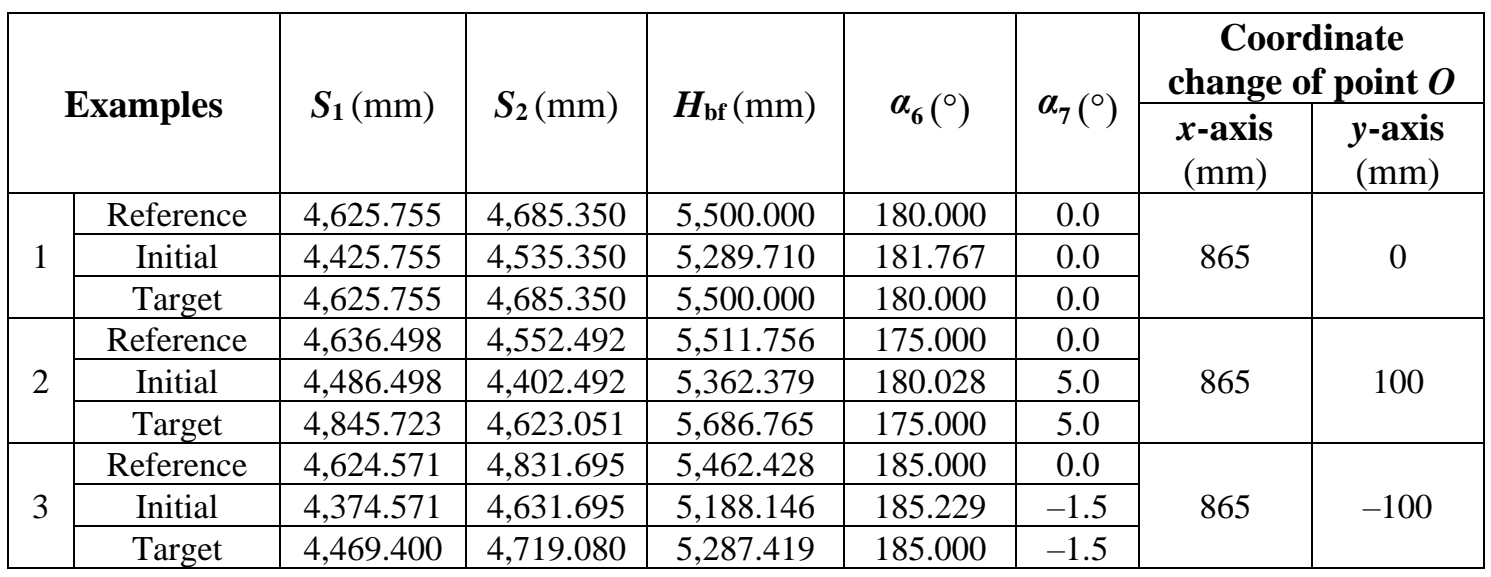

\section{RESULTS AND DISCUSSION}

The m-file compiled through MATLAB is used to simulate three conditions, and the results are shown in Figs. 8, 9 and 10. Figs. 8 a, 9 a and 10 a show the simulation results of the front and rear legs being supplied with the liquid simultaneously, albeit without cooperative control. Figs. $8 \mathrm{~b}, 9 \mathrm{~b}$ and $10 \mathrm{~b}$ show the simulation results of the front and rear legs when using the cooperative control. Figs. $8 \mathrm{c}, 9 \mathrm{c}$ and $10 \mathrm{c}$ show the enlarged view of the rectangular boxes in Figs. 8 b, $9 \mathrm{~b}$ and $10 \mathrm{~b}$, respectively. Figs. $8 \mathrm{~d}, 9 \mathrm{~d}$ and $10 \mathrm{~d}$ show the frequency for the same length of the rear leg when using cooperative control. The values of $y_{\mathrm{R} \max }$ and $y_{\mathrm{Nmax}}$ in Figs. $8 \mathrm{a}$ and $\mathrm{b}$ are the same, and the corresponding curves coincide. Moreover, the values of $y_{\mathrm{R}}$ and $y_{\mathrm{N}}$ in Figs. $9 \mathrm{a}$ and $9 \mathrm{~b}$ are similar, and the corresponding curves are nearly coincident.

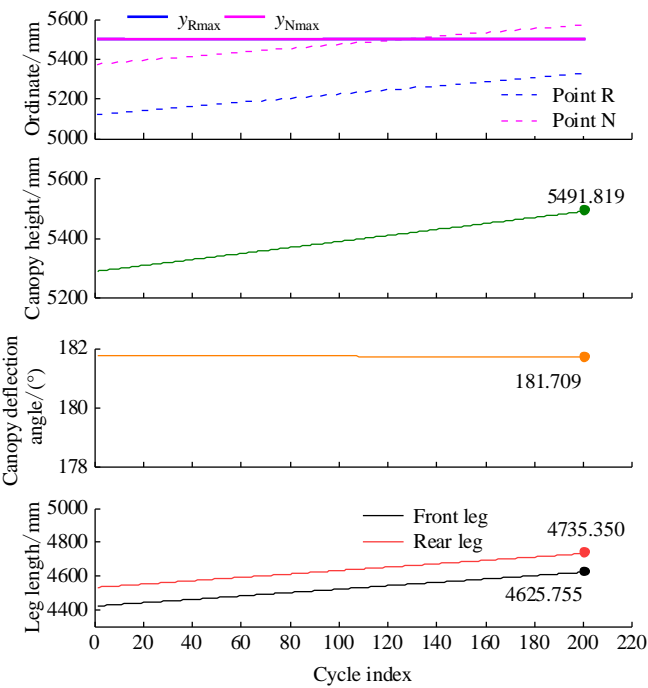

a)

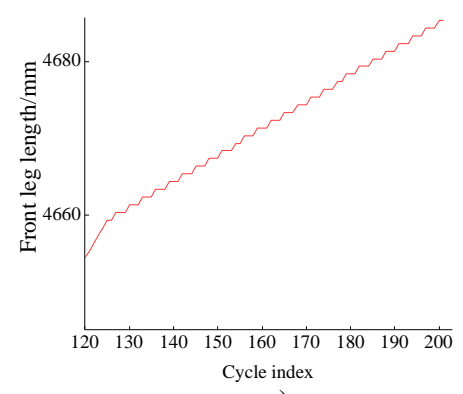

c)

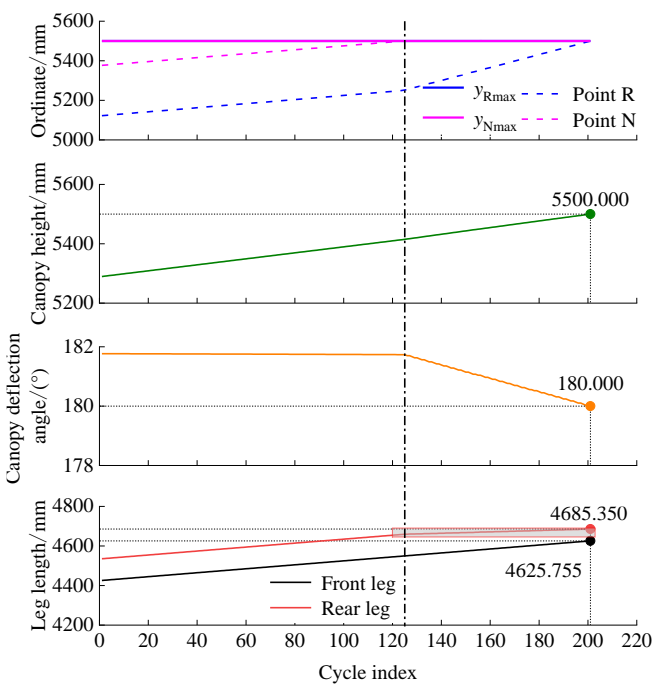

b)

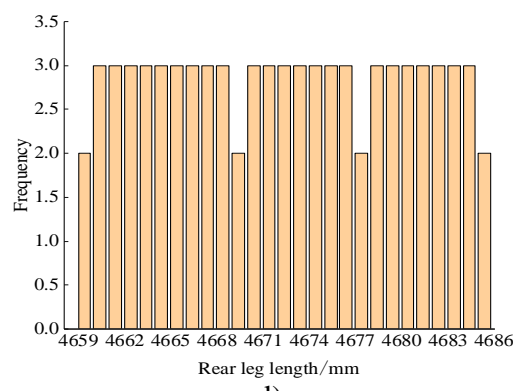

d)

Figure 8: Condition one. 


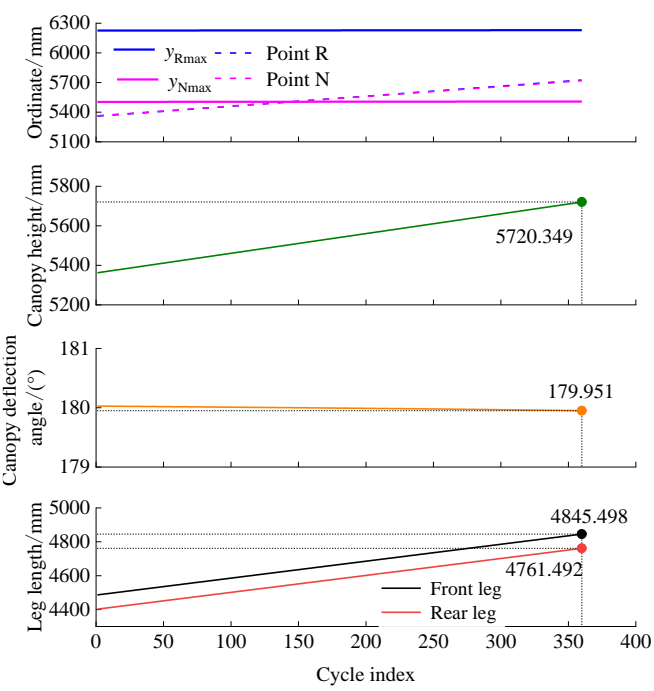

a)

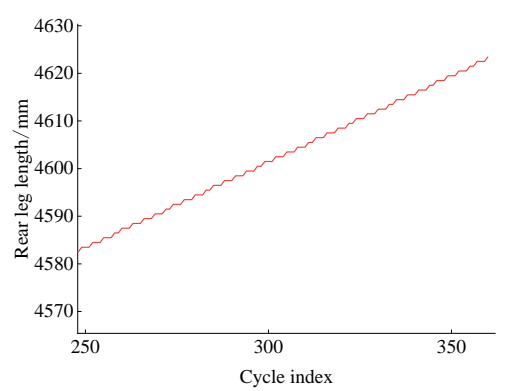

c)

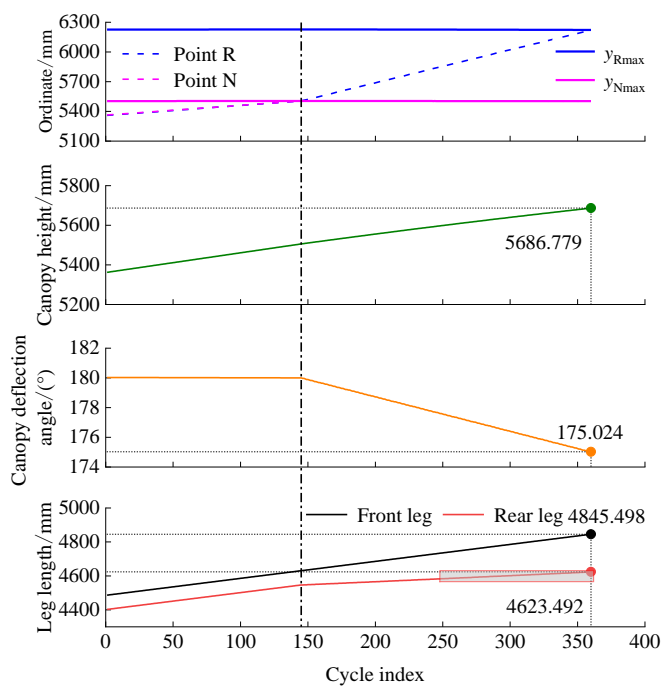

b)

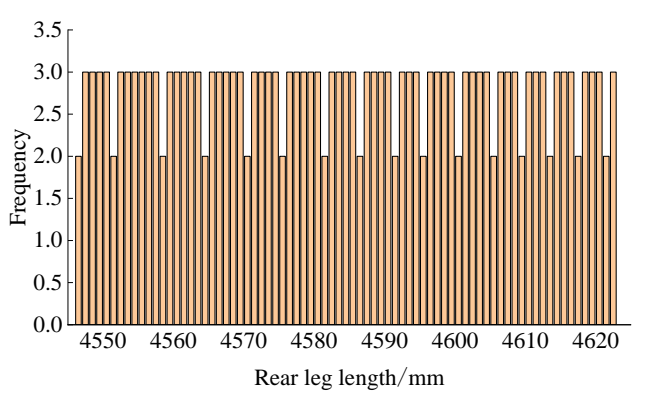

d)

Figure 9: Condition two.

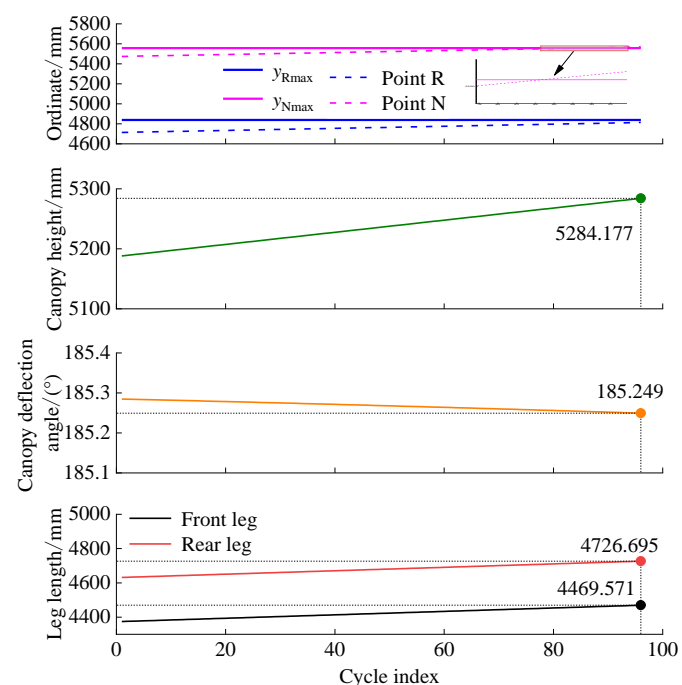

a)

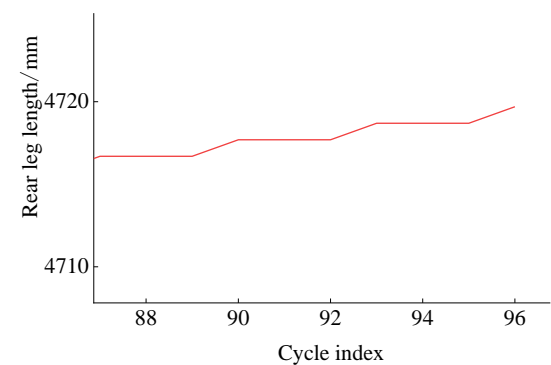

c)

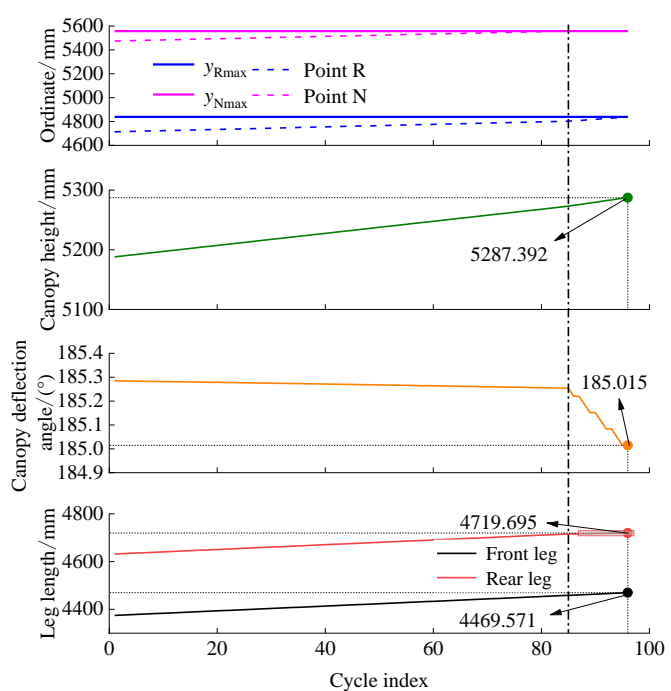

b)

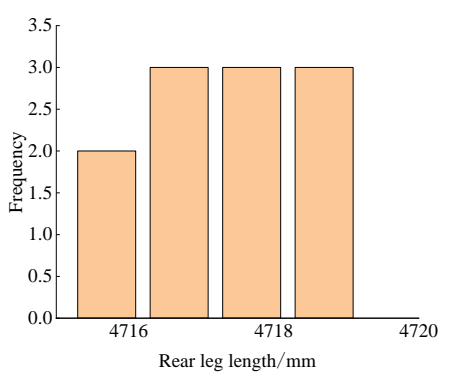

d)

Figure 10: Condition three. 
Comparing Figs. 8 a, 9 a, and 10 a with Figs. 8 b, 9 b, and 10 b, respectively, it can be noted that when the front and rear legs do not employ cooperative control, the rear leg length, canopy deflection angle and canopy height do not approach the target value, and the rear toe of the canopy does not satisfy the constraint conditions. The front and rear leg lengths, canopy height, and front and rear toes of the canopy change linearly. The canopy deflection angle changes according to the regression parabola. The adjusted $R$-square values are listed in Table III. When the coordinated control is adopted, not only do the rear leg length, canopy deflection angle and canopy height approach the target value, the front and rear toes of the canopy do not contact the floor prematurely during the rising process. In addition, although the front leg length changes linearly, the rear leg length, canopy deflection angle, canopy height, and front and rear toes of the canopy exhibit nonlinear changes. In particular, the canopy height exhibits a quintic curve change characteristic.

Table III: Adjusted $R$-square.

\begin{tabular}{|c|c|c|c|}
\hline \multicolumn{2}{|r|}{ Curve } & Adj. $R$-square & Fitting method \\
\hline \multirow{6}{*}{ Fig. 8 a } & Front leg & 1 & Liner fitting \\
\hline & Rear leg & 1 & Liner fitting \\
\hline & Canopy deflection angle & 1 & Cubic polynomial fitting \\
\hline & Canopy height & 1 & Liner fitting \\
\hline & Front toe of canopy & 1 & Liner fitting \\
\hline & Rear toe of canopy & 1 & Liner fitting \\
\hline Fig. $8 \mathrm{~b}$ & Canopy height & 0.999 & Quintic polynomial fitting \\
\hline \multirow{6}{*}{ Fig. 9 a } & Front leg & 1 & Liner fitting \\
\hline & Rear leg & 1 & Liner fitting \\
\hline & Canopy deflection angle & 1 & Cubic polynomial fitting \\
\hline & Canopy height & 1 & Liner fitting \\
\hline & Front toe of canopy & 1 & Liner fitting \\
\hline & Rear toe of canopy & 1 & Liner fitting \\
\hline Fig. $9 \mathrm{~b}$ & Canopy height & 1 & Quintic polynomial fitting \\
\hline \multirow{6}{*}{ Fig. $10 \mathrm{a}$} & Front leg & 1 & Liner fitting \\
\hline & Rear leg & 1 & Liner fitting \\
\hline & Canopy deflection angle & 1 & Cubic polynomial fitting \\
\hline & Canopy height & 1 & Liner fitting \\
\hline & Front toe of canopy & 1 & Liner fitting \\
\hline & Rear toe of canopy & 1 & Liner fitting \\
\hline Fig. $10 \mathrm{~b}$ & Canopy height & 0.999 & Quintic polynomial fitting \\
\hline
\end{tabular}

The dash-dot line perpendicular to the $\mathrm{x}$-axis in Figs. $8 \mathrm{~b}, 9 \mathrm{~b}$ and $10 \mathrm{~b}$ is the line dividing the front and rear leg coordinated control modes. On the left side of this line, the front and rear legs exhibit a synchronous coordinated control mode, which corresponds to the simultaneous rise of the front and rear legs during the non-cooperative control. On the right side of the line, the front and rear legs exhibit a non-synchronous coordinated control mode. According to Figs. $8 \mathrm{c}, 9 \mathrm{c}$ and $10 \mathrm{c}$, the front and rear legs elongate linearly during the synchronous coordinated control mode. In contrast, in the non-synchronous coordinated control mode, although the front leg elongates linearly, the rear leg elongates in a step-like manner, and the elongation of the rear leg is more gradual than that of the front leg. It can be noted from Figs. $8 \mathrm{~d}, 9 \mathrm{~d}$ and $10 \mathrm{~d}$ that in the synchronous coordinated control mode, the front and rear legs are elongated synchronously with a ratio of $1: 1$, whereas in the non-synchronous coordinated control mode, the front and rear legs are elongated in the ratio $2: 1$ or $3: 1$.

In addition, the cycle index is the largest and smallest in conditions two and three, respectively, as the initial value for condition two deviates the most from the target value. 


\section{CONCLUSION}

In this study, a cooperative double closed-loop automatic control technique for the canopy posture was developed, to examine the canopy posture control during the rising process. The canopy posture of three types of roof conditions was simulated using MATLAB, and the front and rear leg length and canopy posture response curves were obtained. According to the research results, the following conclusions could be derived:

(1) When using the proposed collaborative control method, the canopy could approach the target posture, and the front and rear toes of the canopy did not contact the floor prematurely during the rising process.

(2) In the rising process, the front and rear legs exhibited a synchronous or non-synchronous coordinated control mode. In the synchronous coordinated control mode, the front and rear legs were elongated in a linear and synchronous manner. In contrast, in the non-synchronous coordinated control mode, the front and rear legs were elongated synergistically in different proportions.

(3) A larger deviation of the initial posture of the canopy from the target posture corresponded to a more frequent collaborative control of the front and rear legs.

The presented findings can guide the automatic posture control of the canopy of hydraulic supports. However, in this work, the impact of the initial posture on the efficiency of the automatic control was not examined. Future work can be aimed at introducing the initial posture of the support as a constraint condition in the proposed control method.

\section{ACKNOWLEDGEMENT}

This work is supported by the National Natural Science Fund of China (Grant No. U1610251) and the National Key Research and Development Program of China (Grant No. 2017YFC0603005).

\section{REFERENCES}

[1] Meng, Z. S.; Zeng, Q. L.; Gao, K. D.; Kong, S.; Liu, P.; Wan, L. R. (2018). Failure analysis of super-large mining height powered support, Engineering Failure Analysis, Vol. 92, 378-391, doi:10.1016/j.engfailanal.2018.04.011

[2] Szurgacz, D.; Brodny, J. (2019). Tests of geometry of the powered roof support section, Energies, Vol. 12, No. 20, Paper 3945, 19 pages, doi:10.3390/en12203945

[3] Zeng, X. T.; Meng, G. Y.; Zhou, J. H. (2018). Analysis on the pose and dynamic response of hydraulic support under dual impact loads, International Journal of Simulation Modelling, Vol. 17, No. 1, 69-80, doi:10.2507/IJSIMM17(1)412

[4] Wu, Z. M.; Sun, Z. G.; Zhang, W. Z.; Chen, Q. (2014). A novel approach for attitude estimation using MEMS inertial sensors, Proceedings of the IEEE Sensors 2014, 1022-1025, doi:10.1109/ICSENS.2014.6985177

[5] Lin, F. Y.; Miao, Z. Q. (2011). Calculating the movement pose of the supporting and shielding hydraulic support, Coal Science and Technology, Vol. 39, No. 4, 97-100, doi:10.13199/ j.cst.2011.04.102.linfy.026

[6] Liang, M. F.; Fang, X. Q.; Li, S.; Wu, G.; Ma, M.; Zhang, Y. G. (2019). A fiber Bragg grating tilt sensor for posture monitoring of hydraulic supports in coal mine working face, Measurement, Vol. 138, 305-313, doi:10.1016/j.measurement.2019.02.060

[7] Cui, K. (2019). Analysis of supporting condition monitoring system of hydraulic support based on multi-sensor correction, Mechanical Management and Development, Vol. 34, No. 10, 152-153, doi:10.16525/j.cnki.cn14-1134/th.2019.10.062

[8] Chen, D. F.; Li, S. B. (2016). Measurement of coal mining height based on hydraulic support structural angle, Journal of China Coal Society, Vol. 41, No. 3, 788-793, doi:10.13225/ j.cnki.jccs.2015.0685 
[9] Asl, H. G.; Han, K. Y. (2019). Investigation on the influence of parameter uncertainties in the position tracking of robot manipulators, Tehnicki glasnik - Technical Journal, Vol. 13, No. 1, 1624, doi:10.31803/tg-20180921100632

[10] Kim, B. S.; Kim, T. G. (2019). Cooperation of simulation and data model for performance analysis of complex systems, International Journal of Simulation Modelling, Vol. 18, No. 4, 608-619, doi:10.2507/IJSIMM18(4)491

[11] Nair, A. S.; Ezhilarasi, D. (2020). Performance analysis of super twisting sliding mode controller by ADAMS-MATLAB co-simulation in lower extremity exoskeleton, International Journal of Precision Engineering and Manufacturing-Green Technology, Vol. 7, 743-754, doi:10.1007/ s40684-020-00202-w

[12] Liu, G. J.; Zhou, Z. C.; Qian, X.; Wu, X. F.; Pang, W. H. (2016). Multidisciplinary design optimization of a swash-plate axial piston pump, Applied Sciences, Vol. 6, No. 12, Paper 399, 14 pages, doi:10.3390/app6120399

[13] Yang, Y.; Mi, Y. Q.; Qin, D. T.; Yuan, A. H.; Li, G. W. (2019). Analysis of the characteristics of electromechanical-hydraulic model of multi-source drive/transmission system based on periodic excitation, Advances in Mechanical Engineering, Vol. 11, No. 1, Paper 168781401881712, 15 pages, doi:10.1177/1687814018817128

[14] Liang, L. C.; Ren, H. W.; Zheng, H. (2018). Analysis on mechanical-hydraulic coupling rigidity characteristics of hydraulic powered support, Coal Science and Technology, Vol. 46, No. 3, 141 147, doi:10.13199/j.cnki.cst.2018.03.024

[15] Zhang, Y.; Zhang, H. Y.; Gao, K. D.; Xu, W. B.; Zeng, Q. L. (2019). New method and experiment for detecting relative position and posture of the hydraulic support, IEEE Access, Vol. 7, 181842181854, doi:10.1109/ACCESS.2019.2958981

[16] Yang, X. J.; Wang, R. F.; Wang, H. F.; Yang, Y. K. (2020). A novel method for measuring pose of hydraulic supports relative to inspection robot using LiDAR, Measurement, Vol. 154, Paper 107452, 11 pages, doi:10.1016/j.measurement.2019.107452

[17] Ren, H. W.; Li, S. S.; Li, X.; Fu, Z. (2019). Simulation analysis of roof beam position and attitude control of hydraulic support, Industry and Mine Automation, Vol. 45, No. 10, 11-16, doi:10.13272/j.issn.1671-251x.17470

[18] Meng, Z. S.; Zeng, Q. L.; Wan, L. R.; Liu, P. (2018). Pose adjusting simulation of hydraulic support based on mechanical-electrical-hydraulic coordination, Tehnicki vjesnik-Technical Gazette, Vol. 25, No. 4, 1110-1118, doi:10.17559/TV-20180306025203

[19] Meng, Z. S.; Zhang, S.; Xie, Y. Y.; Zeng, Q. L. (2020). Attitude adjustment of backfilling support based on mechanical-hydraulic co-simulation, International Journal of Simulation Modelling, Vol. 19, No. 3, 399-409, doi:10.2507/IJSIMM19-3-520

[20] Xie, J. C.; Wang, X. W.; Yang, Z. J.; Hao, S. Q. (2019). Attitude-aware method for hydraulic support groups in a virtual reality environment, Proceedings of the Institution of Mechanical Engineers, Part C: Journal of Mechanical Engineering Science, Vol. 233, No. 14, 4805-4818, doi: $10.1177 / 0954406219838574$

[21] Ge, X.; Xie, J. C.; Wang, X. W.; Liu, Y.; Shi, H. B. (2020). A virtual adjustment method and experimental study of the support attitude of hydraulic support groups in propulsion state, Measurement, Vol. 158, Paper 107743, 13 pages, doi:10.1016/j.measurement.2020.107743

[22] Wang, F. (2017). Research of precise pushing control scheme for hydraulic support and its application, Industry and Mine Automation, Vol. 43, No. 5, 6-9, doi:10.13272/j.issn.1671$\underline{251 x .2017 .05 .002}$ 